\title{
RECONSTRUCTION OF UNKNOWN CHARACTERISTICS IN A THIRD-ORDER SYSTEM
}

\author{
K. Keesman and V. I. Maksimov
}

\begin{abstract}
The article considers dynamic identification of unknown characteristics in a third-order system. A realtime algorithm is proposed for the solution of the problem. The algorithm relies on constructs from stable dynamical inversion theory, which combines methods of the theory of ill-posed problems and positional control theory. In the proposed procedure, the reconstruction algorithm is represented as a control algorithm for some artificial dynamical system - a model. The model control is adapted to current observations so that its realization eventually "approximates" the unknown input.
\end{abstract}

\section{Introduction}

We consider the problem of reconstructing the unknown characteristics in a third-order system of nonlinear differential equations describing processes in a bioreactor with refeeding. A computer algorithm is proposed for the solution of this problem.

The problem considered in this article belongs to the class of inverse problems of control system dynamics and in a more general context it fits into the framework of ill-posed problems [1,2]. The theory of such problems has been developed with sufficient detail, but only in a posterior setting — without requiring a dynamic reconstruction algorithm [3]. The requirement of dynamism — a special feature of our approach — emerges in situations when current input values are used to make real-time decisions.

The procedure described in this article follows the theory of stable dynamical inversion developed in [4-7]. The latter in turn combines the methods of the theory of ill-posed problems $[1,2]$ and positional control theory [8]. The essence of this theory is that the reconstruction algorithm is represented as a control algorithm for some auxiliary dynamical system - a model; the output of this algorithm in particular is a realization of the model control and it is by definition a dynamic algorithm. Model control is adapted to the results of current observations so that its realization eventually satisfies the conditions of some regularization principle; this ensures that the algorithm is stable. The model control algorithm proposed below is based on a modification of the smoothing functional principle, using an appropriate Lyapunov functional. Model control is constructed so as to ensure slow increase of the functional.

\section{Dynamical Model of a Bioreactor with Refeeding}

The substantive example investigated in this article is derived from $[9,10]$, which focus on optimal experimental design for bioreactor modeling. The present article may be regarded as a continuation of $[9,10]$.

Without loss of generality, we assume that the dissolved oxygen concentration in the refeed is equal to the saturation level, i.e., it is not affected by the bacteria, which by assumption are absent from the refeed. The consumption of the substrate by the bacteria in the reactor is aerobic and directly affects the dissolved oxygen

Ural State University, Yekaterinburg, Russia

Translated from Nelineinaya Dinamika i Upravlenie, No. 7, pp. 149-166, 2010. 
concentration in the reactor. The following nonlinear dynamical model describes the biochemical processes in the reactor [9-11]:

$$
\begin{gathered}
\frac{d C_{D O}(t)}{d t}=k_{L a}\left(C_{\mathrm{sat}}^{\mathrm{en}}-C_{D O}(t)\right)-O U R(t)+\frac{F_{\mathrm{in}}(t)}{V(t)}\left(C_{\mathrm{sat}}-C_{D O}(t)\right), \\
\frac{d C_{X}(t)}{d t}=\mu\left(C_{S}(t)\right) C_{X}(t)-\frac{F_{\text {in }}(t)}{V(t)} C_{X}(t), \\
\frac{d C_{S}(t)}{d t}=-\frac{\mu\left(C_{S}(t)\right)}{Y} C_{X}(t)+\frac{F_{\text {in }}(t)}{V(t)}\left(C_{S, \text { in }}(t)-C_{S}(t)\right),
\end{gathered}
$$

where

$$
\begin{gathered}
V(t)=\int_{0}^{t} F_{\text {in }}(\tau) d \tau+V\left(t_{0}\right), \\
O U R(t)=\frac{(1-Y)}{Y} \mu\left(C_{S}(t)\right) C_{X}(t), \\
\mu\left(C_{S}(t)\right)=\mu_{\max }(t) \frac{C_{S}(t)}{K_{S}+C_{S}(t)}, \quad t \in T=\left[t_{0}, \vartheta\right] .
\end{gathered}
$$

Here and in what follows $k_{L a}$ is the aeration coefficient, $V(t)$ is the reactor volume, $C_{\mathrm{sat}}^{\mathrm{en}}$ is the dissolved oxygen concentration at the saturation level, including a small (constant) correction for endogenous respiration of the biomass, $C_{\text {sat }}$ is the (normal) dissolved oxygen concentration at the saturation level in the refeed, $\mu_{\max }(t)$ is the maximum growth rate, $K_{S}$ is the semi-saturation constant, $Y$ is the coefficient of biomass fluidity on the substrate, $O U R(t)$ is the rate of oxygen absorption by the biomass in the reactor, $C_{D O}(t)$ is the dissolved oxygen concentration in the reactor, $C_{X}(t)$ is the biomass concentration, $C_{S}(t)$ is the biomass growth rate. Note that in (1) the parameter $\mu_{\max }(t)$ is time-dependent. This parameter usually changes due to the adaptation of the organisms, additional constraints on the substrate, or more generally due to kinetic modeling errors.

Note that the first equation in (1) describes the dissolved oxygen concentration in the reactor; the first term in the right-hand side is the natural aeration, the second term is the absorption of oxygen by the aerobic biomass, and the last term is the refeeding and dilution of the dissolved oxygen. The second equation in (1) describes the biomass dynamics; here the first term in the right-hand side describes biomass growth, and the last term describes dilution. The first term responsible for growth is also present - with a certain fluidity coefficient $(Y)-$ in the third equation in (1), but here it characterizes the absorption component in the substrate balance.

In what follows we assume that data are available only for the dissolved oxygen and there is no information about the biomass or the substrate. The limited information capacity is responsible for the fact that the reconstruction of $\mu_{\max }(t)$ and the states $C_{X}(t), C_{S}(t)$ is an ill-posed problem. In what follows we mainly focus on simultaneous estimation of the parameter $\mu_{\max }(t)$, the biomass concentration $C_{X}(t)$, and the biomass growth rate $C_{S}(t)$ using observations with errors of dissolved oxygen concentration $C_{D O}(t)$. We accordingly assume that the parameters $V\left(t_{0}\right), Y, K_{S}, C_{X}\left(t_{0}\right), C_{S}\left(t_{0}\right), C_{\text {sat }}^{\text {en }}, C_{\text {sat }}, k_{L a}$ and the functions $F_{\text {in }}(t), C_{S, \text { in }}(t)$ either have been estimated a priori or are borrowed from the available literature. 


\section{Statement of the Problem and Description of the Algorithm}

The problem considered in this article can be described as follows. The unknown function (coefficient) $\mu_{\max }(t)$ acts on the system (1) generating some (unknown) solution

$$
C(t)=C\left(t ; C_{0}, \mu_{\max }\right)=\left(C_{D O}(t), C_{X}(t), C_{S}(t)\right)
$$

where

$$
C_{0}=\left(C_{D O}\left(t_{0}\right), C_{X}\left(t_{0}\right), C_{S}\left(t_{0}\right)\right)
$$

is the initial system state (assumed known). The time interval $T$ is partitioned into subintervals $\left[\tau_{i}, \tau_{i+1}\right), \tau_{i+1}=$ $\tau_{i}+\delta, \delta>0, i \in[0: m], \tau_{0}=t_{0}, \tau_{m}=\vartheta$. The coordinate $C_{D O}\left(\tau_{i}\right)$ is observed with an error at discrete (sufficiently frequent) time instants $\tau_{i}$; the observations produce the values $\xi_{i}^{h}=\xi\left(\tau_{i}\right) \in \mathbb{R}$ such that

$$
\left|C_{D O}\left(\tau_{i}\right)-\xi_{i}^{h}\right| \leq h
$$

for all $i=1, \ldots, m$. Here and in what follows, $|x|$ stands for the absolute value of the number $a$. It is required to design an algorithm that computes the functions $v(t)=v^{h}(t)$ and $w^{h}(t)=\left\{w_{1}^{h}(t), w_{2}^{h}(t)\right\}$ approximating $\mu_{\max }(t)$ and $C_{X}(t), C_{S}(t)$ respectively.

In what follows, we assume that the number $K \in(0,+\infty)$ is given such that the unknown functions $\mu_{\max }(t)$ and $O U R(t)$ are constrained as follows:

$$
\text { OUR }(t), \quad \mu_{\max }(t) \in L_{\infty}(T ; \mathbb{R}), \quad|O U R(t)| \leq K \quad \text { for almost all } \quad t \in T .
$$

We assume that the following condition holds.

Condition 1. $b_{0} \leq F_{\text {in }}(t) \leq b_{1}$ for almost all $t \in T, 0 \leq b_{0} \leq b_{1}$,

$$
\begin{gathered}
Y \in(0,1), \quad V(t) \geq V_{0}>0, \\
C_{S, \text { in }}(t) \in C^{1}(T ; \mathbb{R}), \quad F_{\text {in }}(t) \in L_{\infty}(T ; \mathbb{R}), \\
C_{X}(t) \geq C_{X}>0, \quad C_{S}(t) \geq C_{S}>0 .
\end{gathered}
$$

Here $\mathbb{R}$ is the set of all real numbers; $C^{1}(T ; \mathbb{R})$ is the space of continuously differentiable functions $x(t)$ : $T \rightarrow \mathbb{R}$ with the norm

$$
\|x(t)\|_{C^{1}}=\max \left\{\max _{t \in T}|x(t)|, \max _{t \in T}|\dot{x}(t)|\right\}
$$

$L_{\infty}(T ; \mathbb{R})$ is the space of Lebesgue measurable functional $x(t): T \rightarrow \mathbb{R}$ with the norm

$$
\|x(t)\|_{L_{\infty}}=\operatorname{vrai} \sup t \in T|x(t)| .
$$

In what follows, the function $V(t)$ is assumed known. 
We solve the problem by the method of auxiliary positionally controlled models developed in [4-7]. Following this method, we first choose and fix the family of partitions

$$
\begin{gathered}
\Delta_{h}=\left\{\tau_{h, i}\right\}_{i=0}^{m_{h}}, \quad \tau_{h, 0}=t_{0}, \quad \tau_{h, m_{h}}=\vartheta, \\
\tau_{h, i+1}=\tau_{h, i}+\delta(h), \quad m_{h}=\left(\vartheta-t_{0}\right) \delta^{-1}(h)
\end{gathered}
$$

of the time interval $T$ with the diameters

$$
\delta(h)=\delta\left(\Delta_{h}\right), \quad \delta(h) \rightarrow 0 \quad \text { as } \quad h \rightarrow 0 .
$$

Here $h$ is the observation error. We then introduce an auxiliary system $M$ functioning synchronously with the real system (1). Note that

(i) $\frac{F_{\text {in }}(t)}{V(t)}=\frac{\dot{V}(t)}{V(t)} \approx \frac{\ln \left(\frac{V\left(\tau_{i+1}\right)}{V\left(\tau_{i}\right)}\right)}{\delta} ;$
(ii) $F_{\text {in }}\left(\tau_{i}\right) \approx \frac{V_{i}-V_{i-1}}{\delta}$.

Therefore, for $M$ we take a discrete linear system of the form

$$
\begin{gathered}
w_{0}^{h}\left(\tau_{i+1}\right)=w_{0}^{h}\left(\tau_{i}\right)+\delta\left\{k_{L a}\left(C_{\mathrm{sat}}^{\mathrm{en}}-\xi_{i}^{h}\right)-\varphi_{i}^{h}\right\}+\ln \left(V\left(\tau_{i+1}\right) V^{-1}\left(\tau_{i}\right)\right)\left(C_{\mathrm{sat}}-\xi_{i}^{h}\right) \\
w_{1}^{h}\left(\tau_{i+1}\right)=W\left(\tau_{i+1}\right) C_{X}\left(t_{0}\right)+V^{-1}\left(\tau_{i+1}\right) \delta \sum_{j=0}^{i} V\left(\tau_{j}\right) \psi_{j}^{h}, \\
w_{2}^{h}\left(\tau_{i+1}\right)=W\left(\tau_{i+1}\right) C_{S}\left(t_{0}\right)+C_{S, \text { in }}\left(\tau_{i+1}\right)-W\left(\tau_{i+1}\right) C_{S, \text { in }}\left(t_{0}\right) \\
-V^{-1}\left(\tau_{i+1}\right)\left[\sum_{j=0}^{i}\left(C_{S, \text { in }}\left(\tau_{j+1}\right)-C_{S, \text { in }}\left(\tau_{j}\right)\right) V\left(\tau_{j}\right)+\delta Y^{-1} \sum_{j=0}^{i} V\left(\tau_{j}\right) \psi_{j}^{h}\right],
\end{gathered}
$$

with the initial condition

$$
w_{0}^{h}\left(t_{0}\right)=\xi_{0}^{h}, \quad w_{1}^{h}\left(t_{0}\right)=C_{X}\left(t_{0}\right), \quad w_{2}^{h}\left(t_{0}\right)=C_{S}\left(t_{0}\right)
$$

Here

$$
\psi_{i}^{h}=\frac{Y \varphi_{i}^{h}}{(1-Y)}, \quad \varphi_{i}^{h}=\varphi^{h}\left(\tau_{i}\right), \quad W\left(\tau_{j}\right)=V^{-1}\left(\tau_{j}\right) V\left(t_{0}\right)
$$

Thus, the input of model $M$ is the control $\varphi^{h}(\cdot)$ and its output is the trajectory $w^{h}(\cdot)$,

$$
\varphi^{h}(t)=\varphi_{i}^{h}, \quad w^{h}(t)=\left\{w_{0}^{h}(t), w_{1}^{h}(t), w_{2}^{h}(t)\right\}=w^{h}\left(\tau_{i}\right), \quad t \in\left[\tau_{i}, \tau_{i+1}\right),
$$


where the vectors $w^{h}\left(\tau_{i}\right)$ are determined from the formulas (5) and the rule for the determination of $\varphi_{i}^{h}$ is given below.

Let us proceed with the description of the algorithm. Prior to the instant $t_{0}$ we fix the value $h \in(0,1)$, the function

$$
\alpha=\alpha(h):(0,1) \rightarrow \mathbb{R}^{+}=\{r \in \mathbb{R}: r>0\}
$$

the partition $\Delta=\Delta_{h}$ (4) with the diameter $\delta=\delta\left(\Delta_{h}\right)$, and the model (5). We then set up a feedback control for model $M$ that evolves synchronously with the functioning of system (1). The algorithm starts at the time $t_{0}$ and is divided into $m_{h}-1$ identical steps. The following operations are performed during step $i$, which takes place on the time interval $\delta_{i}=\left[\tau_{i}, \tau_{i+1}\right)$. First we find the model control

$$
\varphi^{h}(t)=\varphi_{i}^{h}, \quad t \in \delta_{i}
$$

with the feedback $(\alpha(h)$ and $K$ are auxiliary parameters)

$$
\varphi_{i}^{h}=\varphi_{i}^{h}\left(\xi_{i}^{h}, w_{0}^{h}\left(\tau_{i}\right)\right)= \begin{cases}-s_{i} \alpha^{-1}(h) & \text { if } \quad\left|s_{i}\right| \leq \alpha(h) K \\ -K \operatorname{sign} s_{i} & \text { otherwise }\end{cases}
$$

$s_{i}=\xi_{i}^{h}-w_{0}^{h}\left(\tau_{i}\right)$. Then we find the phase state $w^{h}\left(\tau_{i+1}\right)$ of the model at time $\tau_{i+1}$ from (5). After that the function $v^{h}(\cdot)$ approximating $\mu_{\max }(\cdot)$ is obtained by the rule

$$
v^{h}(t)=v_{i}^{h}, \quad t \in\left[\tau_{i}, \tau_{i+1}\right),
$$

where

$$
v_{i}^{h}=v_{i}^{h}\left(\xi_{i}^{h}, \varphi_{i}^{h}, w^{h}\left(\tau_{i}\right)\right)=\frac{Y\left(K_{S}+w_{2}^{h}\left(\tau_{i}\right)\right) \varphi_{i}^{h}}{(1-Y) w_{1}^{h}\left(\tau_{i}\right) w_{2}^{h}\left(\tau_{i}\right)} .
$$

The algorithm stops at time $\vartheta$.

The convergence of the algorithm is established by Theorem 1 (see below). We introduce the following condition.

Condition 2. There are numbers $w_{1}>0$ and $w_{2}>0$ such that for all $h \in(0,1)$ and $t \in T$ we have the inequalities

$$
w_{1}^{h}(t) \geq w_{1}, \quad w_{2}^{h}(t) \geq w_{2} .
$$

We thus have the following theorem.

Theorem 1. Assume that Conditions 1 and 2 hold, as well as the following matching conditions for the algorithm parameters:

$$
\alpha(h) \rightarrow 0, \quad(h+\delta(h)) \alpha^{-1}(h) \rightarrow 0 \quad \text { as } \quad h \rightarrow 0 .
$$


Then we have the convergences

$$
\begin{gathered}
\sup _{t \in T}\left|w_{1}^{h}(t)-C_{X}(t)\right| \rightarrow 0, \quad \sup _{t \in T}\left|w_{2}^{h}(t)-C_{S}(t)\right| \rightarrow 0, \\
\int_{t_{0}}^{\vartheta}\left|v^{h}(\tau)-\mu_{\max }(\tau)\right|^{2} d \tau \rightarrow 0 \quad \text { as } \quad h \rightarrow 0 .
\end{gathered}
$$

We see from this theorem that the function

$$
v^{h}(t)=v^{h}\left(\xi(t), \varphi^{h}(t), w^{h}(t)\right)
$$

may be treated as an "approximation" of the unknown variable coefficient $\mu_{\max }(t)$ for the corresponding $h$, and the functions $w_{1}^{h}(t), w_{2}^{h}(t)$ are approximations of $C_{X}(t)$ and $C_{S}(t)$, respectively.

\section{Proof of Convergence of the Algorithm}

Before proceeding to prove Theorem 1, we present some auxiliary bounds.

Let

$$
c^{(0)}=2 F_{\max }\left(C_{S, \text { in }}\left(t_{0}\right)+C_{\max }\left(\vartheta-t_{0}\right)\right)
$$

and

$$
i(t)=\kappa\left(\left(t-t_{0}\right) / \delta\right)
$$

where $\kappa(a)$ is the whole part of $a$. Define

$$
a(t)=\frac{d(\ln V(t))}{d t}
$$

Lemma 1. Let

$$
\left|\dot{C}_{S, \text { in }}(t)\right| \leq C_{\max } \quad \text { and } \quad 0<F_{\text {in }}(t) \leq F_{\max }
$$

for almost all $t \in T$. Then we have the inequality

$$
\begin{aligned}
\mid \int_{t_{0}}^{t} V(\tau) a(\tau) C_{S, \text { in }}(\tau) d \tau- & {\left[C_{S, \text { in }}\left(\tau_{i(t)}\right) V\left(\tau_{i(t)}\right)-C_{S, \text { in }}\left(t_{0}\right) V\left(t_{0}\right)\right.} \\
& \left.-\sum_{i=0}^{i(t)-1}\left(C_{S, \text { in }}\left(\tau_{i+1}\right)-C_{S, \text { in }}\left(\tau_{i}\right)\right) V\left(\tau_{i}\right)\right] \mid \leq c^{(0)} \delta \quad \text { for all } t \in T .
\end{aligned}
$$


Proof. From the inequality

$$
|V(t)-V(t+\delta)| \leq \int_{t}^{t+\delta}\left|F_{\text {in }}(\tau)\right| d \tau \leq F_{\max } \delta, \quad t, t+\delta \in T
$$

we obtain the bound

$$
\left|\int_{t_{0}}^{t} \dot{C}_{S, \text { in }}(\tau)\left(V(\tau)-V_{\delta}(\tau)\right) d \tau\right| \leq F_{\max }\left(t-t_{0}\right) C_{\max } \delta
$$

where $V_{\delta}(t)=V\left(\tau_{i}\right)$ for $t \in\left[\tau_{i}, \tau_{i+1}\right)$. Note that

$$
\int_{t}^{t+\delta}\left|V(\tau) a(\tau) C_{S, \text { in }}(\tau)\right| d \tau \leq F_{\max }\left(C_{S, \text { in }}\left(t_{0}\right)+C_{\max }\left(\vartheta-t_{0}\right)\right) \delta .
$$

Therefore for $t \in\left[\tau_{i}, \tau_{i+1}\right)$ we have

$$
\left|\int_{t_{0}}^{\tau_{i}} V(\tau) a(\tau) C_{S, \text { in }}(\tau) d \tau-\int_{t_{0}}^{t} V(\tau) a(\tau) C_{S, \text { in }}(\tau) d \tau\right| \leq F_{\max }\left(C_{S, \text { in }}\left(t_{0}\right)+C_{\max }\left(\vartheta-t_{0}\right)\right) \delta .
$$

Integrating by parts, we obtain

$$
\int_{t_{0}}^{\tau_{i}} V(\tau) a(\tau) C_{S, \text { in }}(\tau) d \tau=V\left(\tau_{i}\right) C_{S, \text { in }}\left(\tau_{i}\right)-V\left(t_{0}\right) C_{S, \text { in }}\left(t_{0}\right)-\int_{t_{0}}^{\tau_{i}} V(\tau) \dot{C}_{S, \text { in }}(\tau) d \tau
$$

The sought inequality follows from (8), (9) and the preceding equality. Q.E.D.

Lemma 2. Assume that the conditions of Lemma 1 hold, $V(t) \geq V_{0}>0$, and

$$
\tilde{b}(\tau)=\tilde{b}_{j} \quad \text { for } \quad \tau \in\left[\tau_{j}, \tau_{j+1}\right), \quad\left|\tilde{b}_{j}\right| \leq d \quad \text { for } \quad j \in\left[0: m_{h}-1\right] .
$$

Then we have the inequality

$$
\left|V^{-1}(t) \int_{t_{0}}^{t} V(\tau) \tilde{b}(\tau) d \tau-V^{-1}\left(\tau_{i(t)}\right) \delta \sum_{j=0}^{i(t)} V\left(\tau_{j}\right) \tilde{b}_{j}\right| \leq c_{1} \delta \quad \text { for } \quad t \in T \text {. }
$$

Lemma 2 is proved by simple algebraic manipulations. 
Introduce a system of ordinary differential equations of second order

$$
\begin{gathered}
\dot{C}_{x \psi}(t)=\psi(t)-a(t) C_{x \psi}(t), \quad t \in T, \\
\dot{C}_{S \psi}(t)=-\frac{\psi(t)}{Y}+a(t) C_{S, \text { in }}(t)-a(t) C_{S \psi}(t)
\end{gathered}
$$

with initial conditions

$$
C_{x \psi}\left(t_{0}\right)=C_{X}\left(t_{0}\right), \quad C_{S \psi}\left(t_{0}\right)=C_{S}\left(t_{0}\right)
$$

and function $\psi(\cdot)$ of the form

$$
\psi(t)=\psi_{j}^{h}=Y \varphi_{j}^{h} /(1-Y) \text { for } t \in\left[\tau_{j}, \tau_{j+1}\right)
$$

Define the piecewise-constant functions

$$
w_{1}^{h}(t)=w_{1}^{h}\left(\tau_{i}\right), \quad w_{2}^{h}(t)=w_{2}^{h}\left(\tau_{i}\right) \quad \text { for } \quad t \in\left[\tau_{i}, \tau_{i+1}\right) \cap T .
$$

Lemma 3. Assume that the conditions of Lemmas 1 and 2 hold. Then we have the inequalities

$$
\left|C_{x \psi}(t)-w_{1}^{h}(t)\right| \leq c_{2} \delta, \quad\left|C_{S \psi}(t)-w_{2}^{h}(t)\right| \leq c_{3} \delta \quad \text { for } \quad t \in T
$$

Proof. Note that the equation

$$
\dot{x}(t)=f(t)-a(t) x(t), \quad x\left(t_{0}\right)=x_{0}, \quad f(\cdot) \in L_{2}(T ; \mathbb{R}),
$$

has a solution, which can be obtained by the Cauchy formula

$$
x(t)=V^{-1}(t) V\left(t_{0}\right) x_{0}+V^{-1}(t) \int_{t_{0}}^{t} V(\tau) f(\tau) d \tau .
$$

Therefore, the solution of system (10) is obtained by the formulas

$$
\begin{gathered}
C_{x \psi}(t)=V^{-1}(t) V\left(t_{0}\right) C_{X}\left(t_{0}\right)+\int_{t_{0}}^{t} V^{-1}(t) V(\tau) \psi(\tau) d \tau, \\
C_{S \psi}(t)=V^{-1}(t) V\left(t_{0}\right) C_{S}\left(t_{0}\right)+\int_{t_{0}}^{t} V^{-1}(t) V(\tau)\left(a(\tau) C_{S, \text { in }}(\tau)-\psi(\tau) Y^{-1}\right) d \tau .
\end{gathered}
$$

The lemma follows from these two inequalities and Lemmas 1 and 2. Q.E.D. 
Lemma 4. Let $Y \in(0,1)$ and assume that the conditions of Lemma 3 hold. Then we have the inequalities

$$
\begin{gathered}
\left|C_{X}(t)-w_{1}^{h}(t)\right| \leq c_{4}\left(\delta+\int_{t_{0}}^{t}\left|\varphi^{h}(\tau)-O U R(\tau)\right| d \tau\right), \\
\left|C_{S}(t)-w_{2}^{h}(t)\right| \leq c_{5}\left(\delta+\int_{t_{0}}^{t}\left|\varphi^{h}(\tau)-O U R(\tau)\right| d \tau\right) \quad \text { for } \quad t \in T .
\end{gathered}
$$

Proof. Let

$$
\mu_{1}(t)=C_{x \psi}(t)-C_{X}(t), \quad \mu_{2}(t)=C_{S \psi}(t)-C_{S}(t)
$$

The second and the third equations in system (1) may be rewritten as

$$
\begin{gathered}
\frac{d C_{X}(t)}{d t}=\frac{Y}{1-Y} O U R(t)-a(t) C_{X}(t) \\
\frac{d C_{S}(t)}{d t}=-\frac{1}{1-Y} O U R(t)+a(t)\left(C_{S, \text { in }}(t)-C_{S}(t)\right) .
\end{gathered}
$$

The functions $\mu_{1}(t)$ and $\mu_{2}(t)$ are thus solutions of the equations

$$
\begin{gathered}
\dot{\mu}_{1}(t)=\frac{Y}{1-Y}\left(\varphi^{h}(t)-O U R(t)\right)-a(t) \mu_{1}(t), \\
\dot{\mu}_{2}(t)=-\frac{1}{1-Y}\left(\varphi^{h}(t)-O U R(t)\right)-a(t) \mu_{2}(t),
\end{gathered}
$$

with initial conditions

$$
\mu_{1}\left(t_{0}\right)=\mu_{2}\left(t_{0}\right)=0
$$

Hence

$$
\begin{aligned}
& \mu_{1}(t)=\frac{Y}{1-Y} \int_{t_{0}}^{t} \Phi(t, \tau)\left(\varphi^{h}(\tau)-O U R(\tau)\right) d \tau, \\
& \mu_{2}(t)=-\frac{1}{1-Y} \int_{t_{0}}^{t} \Phi(t, \tau)\left(\varphi^{h}(\tau)-O U R(\tau)\right) d \tau,
\end{aligned}
$$

where

$$
\Phi(t, \tau)=V^{-1}(t) V(\tau) .
$$


In this case, we have the inequalities

$$
\begin{gathered}
\left|\mu_{1}(t)\right| \leq c_{1} \int_{t_{0}}^{t}\left|\varphi^{h}(\tau)-O U R(\tau)\right| d \tau, \\
\left|\mu_{2}(t)\right| \leq c_{2} \int_{t_{0}}^{t}\left|\varphi^{h}(\tau)-O U R(\tau)\right| d \tau, \quad t \in T .
\end{gathered}
$$

The lemma follows from (12), (13) and Lemma 3.

Lemma 5. Assume that the conditions of Lemma 4 hold and

$$
C_{X}(t) \geq C_{X}>0, \quad C_{S}(t) \geq C_{S}>0 .
$$

Then, if Condition 2 is satisfied, we can find $h_{*}>0$ such that for all $h h \in\left(0, h_{*}\right)$ and all $t \in\left[\tau_{i}, \tau_{i+1}\right)$, $i \in\left[0: m_{h}-1\right]$ we have the inequality

$$
\left|v_{i}^{h}-\mu_{\max }(t)\right| \leq c_{6}\left(\delta+\left|\varphi_{i}^{h}-O U R(t)\right|+\int_{t_{0}}^{t}\left|\varphi^{h}(\tau)-O U R(\tau)\right| d \tau\right)
$$

Proof. Applying Lemma 2 and Condition 2, we can find $h_{1}$ and $\delta_{1}$ such that for all $h \in\left(0, h_{1}\right)$ and $\delta=\delta(h) \in\left(0, \delta_{1}\right)$ the following inequalities hold:

$$
C_{x \psi(t)} \geq c_{\psi}^{(1)}>0, \quad C_{S \psi}(t) \geq c_{\psi}^{(2)}>0 \quad \text { for } \quad t \in T
$$

Let

$$
\Delta_{i, t}^{h} \equiv\left|\frac{Y\left(K_{S}+w_{2}^{h}\left(\tau_{i}\right)\right) \varphi_{i}^{h}}{(1-Y) w_{1}^{h}\left(\tau_{i}\right) w_{2}^{h}\left(\tau_{i}\right)}-\frac{Y\left(K_{S}+C_{S \psi}(t)\right) O U R(t)}{(1-Y) C_{x \psi}(t) C_{S \psi}(t)}\right|
$$

By Condition 2, inequalities (15), and Lemma 3, we have the bounds

$$
\Delta_{i, t}^{h} \leq c_{3}\left(\delta+\left|\varphi_{i}^{h}-O U R(t)\right|\right), \quad t \in\left[\tau_{i}, \tau_{i+1}\right)
$$

uniformly in all $i \in\left[0: m_{h}-1\right]$ and $h \in\left(0, h_{1}\right)$. Furthermore, by (12), (13) we have the inequalities

$$
\begin{gathered}
\left|\frac{1}{C_{X}(t) C_{S}(t)}-\frac{1}{C_{x \psi}(t) C_{S \psi}(t)}\right| \leq c_{4}\left(\left|\mu_{1}(t)\right|+\left|\mu_{2}(t)\right|\right) \leq c_{5} \int_{t_{0}}^{t}\left|\varphi^{h}(\tau)-O U R(\tau)\right| d \tau, \\
\qquad\left|\frac{1}{C_{X}(t)}-\frac{1}{C_{x \psi}(t)}\right| \leq c_{6} \int_{t_{0}}^{t}\left|\varphi^{h}(\tau)-O U R(\tau)\right| d \tau,
\end{gathered}
$$


From (18), (19) we obtain

$$
\Delta_{t} \equiv\left|\frac{K_{S}+C_{S \psi}(t)}{C_{x \psi}(t) C_{S \psi}(t)}-\frac{K_{S}+C_{S}(t)}{C_{X}(t) C_{S}(t)}\right| \leq c_{7} \int_{t_{0}}^{t}\left|\varphi^{h}(\tau)-O U R(\tau)\right| d \tau
$$

Moreover,

$$
\begin{gathered}
\mu_{\max }(t)=\frac{\mu\left(C_{S}(t)\right)\left(K_{S}+C_{S}(t)\right)}{C_{S}(t)}, \\
\mu\left(C_{S}(t)\right)=\frac{Y \cdot O U R(t)}{(1-Y) C_{X}(t)} .
\end{gathered}
$$

Thus,

$$
\mu_{\max }(t)=\frac{Y\left(K_{S}+C_{S}(t)\right) O U R(t)}{(1-Y) C_{X}(t) C_{S}(t)}
$$

and

$$
\left|v_{i}^{h}-\mu_{\max }(t)\right|=\left|\frac{Y\left(K_{S}+w_{2}^{h}\left(\tau_{i}\right)\right) \varphi_{i}^{h}}{(1-Y) w_{1}^{h}\left(\tau_{i}\right) w_{2}^{h}\left(\tau_{i}\right)}-\frac{Y\left(K_{S}+C_{S}(t)\right) O U R(t)}{(1-Y) C_{X}(t) C_{S}(t)}\right| .
$$

Combining (17), (20) and applying the inequality

$$
\left|v_{i}^{h}-\mu_{\max }(t)\right| \leq \Delta_{i, t}^{h}+\frac{Y}{1-Y}|O U R(t)| \Delta_{t}, \quad t \in\left[\tau_{i}, \tau_{i+1}\right), \quad i \in\left[0: m_{h}-1\right],
$$

we obtain (14). Q.E.D.

Lemma 6. Assume that Conditions 1 and 2 hold and that $\varphi_{j}^{h}$ in system (5) are obtained from formulas (6). Then we have the inequalities

$$
\begin{gathered}
\left|w_{0}^{h}\left(\tau_{i}\right)-C_{D O}\left(\tau_{i}\right)\right|^{2} \leq C^{(0)}(h+\delta+\alpha), \\
\int_{t_{0}}^{\vartheta}\left|\varphi^{h}(\tau)\right|^{2} d \tau \leq \int_{t_{0}}^{\vartheta}|O U R(\tau)|^{2} d \tau+C^{(1)}(h+\delta) \alpha^{-1} .
\end{gathered}
$$

Proof. To prove the lemma, we need a bound on the increments of

$$
\varepsilon(t)=\left|\tilde{w}_{0}^{h}(t)-C_{D O}(t)\right|^{2}+\alpha(h) \int_{t_{0}}^{t}\left\{\left|\varphi^{h}(\tau)\right|^{2}-|O U R(\tau)|^{2}\right\} d \tau, \quad t \in T .
$$


Here the function $\tilde{w}_{0}^{h}(t), t \in\left[\tau_{i}, \tau_{i+1}\right), i \in\left[0: m_{h}-1\right]$ is defined by the rule

$$
\begin{gathered}
\dot{\tilde{w}}_{0}^{h}(t)=k_{L a}\left(C_{\text {sat }}^{\mathrm{en}}-\xi_{i}^{h}\right)-\varphi_{i}^{h}+a(t)\left(C_{\text {sat }}-\xi_{i}^{h}\right), \quad t \in\left[\tau_{i}, \tau_{i+1}\right), \\
\dot{\tilde{w}}_{0}^{h}\left(\tau_{i}\right)=w_{0}^{h}\left(\tau_{i}\right) .
\end{gathered}
$$

Note that

$$
\lim _{t \rightarrow \tau_{i+1}-0} \tilde{w}_{0}^{h}(t)=w_{0}^{h}\left(\tau_{i+1}\right)
$$

Let

$$
\mu_{i}=2\left(\tilde{w}_{0}^{h}\left(\tau_{i}\right)-C_{D O}\left(\tau_{i}\right)\right) \int_{\tau_{i}}^{\tau_{i+1}}\left(\dot{\tilde{w}}_{0}^{h}(t)-\dot{C}_{D O}(t)\right) d t
$$

It is easy to see that we have the inequality

$$
\varepsilon\left(\tau_{i+1}\right) \leq \varepsilon\left(\tau_{i}\right)+\delta(h) \int_{\tau_{i}}^{\tau_{i+1}}\left|\dot{\tilde{w}}_{0}^{h}(\tau)-\dot{C}_{D O}(\tau)\right|^{2} d \tau+\mu_{i}+\alpha(h) \int_{\tau_{i}}^{\tau_{i+1}}\left\{\left|\varphi_{i}^{h}\right|^{2}-|O U R(\tau)|^{2}\right\} d \tau .
$$

Consider the quantity $\mu_{i}$ in the right-hand side of (23). We have

$$
\mu_{i}=-2 s_{i}^{*} \int_{\tau_{i}}^{\tau_{i+1}}\left\{k_{L a}\left(C_{D O}(\tau)-\xi_{i}^{h}\right)+a(\tau)\left(C_{D O}(\tau)-\xi_{i}^{h}\right)+O U R(\tau)-\varphi_{i}^{h}\right\} d \tau=\sum_{j=1}^{3} \lambda_{j i}
$$

where

$$
\begin{gathered}
\lambda_{1 i}=2 k_{L a} s_{i}^{*} \int_{\tau_{i}}^{\tau_{i+1}}\left(\xi_{i}^{h}-C_{D O}(\tau)\right) d \tau, \quad \lambda_{2 i}=2 s_{i}^{*} \int_{\tau_{i}}^{\tau_{i+1}} a(\tau)\left(\xi_{i}^{h}-C_{D O}(\tau)\right) d \tau \\
\lambda_{3 i}=2 s_{i}^{*} \int_{\tau_{i}}^{\tau_{i+1}}\left(\varphi_{i}^{h}-O U R(\tau)\right) d \tau, \quad s_{i}^{*}=C_{D O}\left(\tau_{i}\right)-w_{0}^{h}\left(\tau_{i}\right) .
\end{gathered}
$$

Therefore, from (23), (24) we obtain

$$
\varepsilon\left(\tau_{i+1}\right) \leq \varepsilon\left(\tau_{i}\right)+\sum_{j=1}^{3} \lambda_{j i}+\alpha(h) \int_{\tau_{i}}^{\tau_{i+1}}\left\{\left|\varphi_{i}^{h}\right|^{2}-|O U R(\tau)|^{2}\right\} d \tau+\delta L_{i}^{h}
$$

where

$$
L_{i}^{h}=\int_{\tau_{i}}^{\tau_{i+1}}\left|k_{L a}\left(\xi_{i}^{h}-C_{D O}(\tau)\right)+a(\tau)\left(\xi_{i}^{h}-C_{D O}(\tau)\right)+\varphi_{i}^{h}-O U R(\tau)\right|^{2} d \tau
$$


Let us bound each term in the right-hand side of inequality (25). From (3) and Condition 1 we have

$$
\max \left\{\sup _{t_{0} \leq t \leq \vartheta}\left|C_{D O}(t)\right|, \sup _{t_{0} \leq t \leq \vartheta}\left|C_{X}(t)\right|, \sup _{t_{0} \leq t \leq \vartheta}\left|C_{S}(t)\right|\right\} \leq d_{0}<+\infty .
$$

From this inequality and the inequality $\left|\xi_{i}^{h}-C_{D O}\left(\tau_{i}\right)\right| \leq h$ we thus obtain

$$
\begin{gathered}
\lambda_{1 i} \leq d_{1}(h+\delta) \delta, \\
\lambda_{2 i} \leq d_{2}(h+\delta) \delta \\
\lambda_{3 i} \leq 2 s_{i} \int_{\tau_{i}}^{\tau_{i+1}}\left(\varphi_{i}^{h}-O U R(\tau)\right) d \tau+d_{3} h \delta, \quad s_{i}=\xi_{i}^{h}-w_{0}^{h}\left(\tau_{i}\right), \\
\sum_{i=0}^{m_{h}-1} L_{i}^{h} \leq d_{4} .
\end{gathered}
$$

Here $d_{j}, j \in[0: 4]$, are constants for which explicit expressions may be written. Note that

$$
\varphi_{i}^{h}=\arg \min \left\{2 s_{i} u+\alpha(h) u^{2}:-K \leq u \leq K\right\}
$$

Then by (3), (6), and (28), we obtain the chain of inequalities

$$
\begin{aligned}
\lambda_{3 i}+ & \alpha(h) \int_{\tau_{i}}^{\tau_{i+1}}\left\{\left|\varphi_{i}^{h}\right|^{2}-|O U R(\tau)|^{2}\right\} d \tau \\
& \leq \int_{\tau_{i}}^{\tau_{i+1}}\left\{\left[2 s_{i} \varphi_{i}^{h}+\alpha(h)\left|\varphi_{i}^{h}\right|^{2}\right]-\left[2 s_{i} O U R(\tau)+\alpha(h)|O U R(\tau)|^{2}\right]\right\} d \tau+d_{3} h \delta \leq d_{3} h \delta .
\end{aligned}
$$

Using (25)-(28), we obtain (for all $i \in\left[1: m_{h}\right]$ ))

$$
\varepsilon\left(\tau_{i}\right) \leq d_{5}(h+\delta)
$$

Inequalities (21), (22) follow from (31). Q.E.D.

Theorem 1.2.1 [5] can be restated in the following form in application to our case.

Theorem 2. Assume that Conditions 1 and 2 hold and also the inequality

$$
\sup _{i \in\left[0: m_{h}\right]}\left|w_{0}^{h}\left(\tau_{i}\right)-C_{D O}\left(\tau_{i}\right)\right| \leq \nu(h),
$$




$$
\int_{t_{0}}^{\vartheta}\left|\varphi^{h}(\tau)\right|^{2} d \tau \leq \int_{t_{0}}^{\vartheta}|O U R(\tau)|^{2} d \tau+\nu_{1}(h),
$$

where $\nu(h) \rightarrow 0, \nu_{1}(h) \rightarrow 0+$ as $h \rightarrow 0+$. Then we have the convergence

$$
\varphi^{h}(t) \rightarrow O U R(t) \quad \text { in } \quad L_{2}(T ; \mathbb{R}) \quad \text { as } \quad h \rightarrow 0
$$

i.e.,

$$
\int_{t_{0}}^{\vartheta}\left|\varphi^{h}(\tau)-O U R(\tau)\right|^{2} d \tau \rightarrow 0 \quad \text { as } \quad h \rightarrow 0 .
$$

Lemmas 4-6 and Theorem 2 imply Theorem 1 . We have thus established convergence of the proposed algorithm.

\section{Bound on the Rate of Convergence of the Algorithm}

We now derive a bound on the rate of convergence of the algorithm.

Theorem 3. Assume that $\mu_{\max }(t)$ is a function of bounded variation.

Then we have the following bounds on the rate of convergence of the algorithm:

$$
\begin{aligned}
& \int_{t_{0}}^{\vartheta}\left|v^{h}(\tau)-\mu_{\max }(\tau)\right|^{2} d \tau \leq C_{1} \lambda(h, \delta, \alpha), \\
& \sup _{t \in T}\left|w_{1}^{h}(t)-C_{X}(t)\right| \leq C_{2} \lambda^{1 / 2}(h, \delta, \alpha), \\
& \sup _{t \in T}\left|w_{2}^{h}(t)-C_{S}(t)\right| \leq C_{3} \lambda^{1 / 2}(h, \delta, \alpha) .
\end{aligned}
$$

Here

$$
\lambda(h, \delta, \alpha)=h+\delta+\alpha+(h+\delta) \alpha^{-1}, \quad C_{j} \quad(j=1,2,3)
$$

are some constants that may written out in explicit form.

Proof. It is easy to see that for all $t \in \delta_{i}=\left[\tau_{i}, \tau_{i+1}\right)$ we have the bounds

$$
\begin{gathered}
\left|\xi_{i}^{h}-C_{D O}(t)\right| \leq c_{1}(h+\delta), \\
\left|C_{D O}\left(t_{0}\right)-w_{0}^{h}\left(t_{0}\right)\right| \leq h, \\
\left|b(t)-b\left(\tau_{i}\right)\right| \leq c_{2} \delta, \quad\left|C_{D O}(t)-C_{D O}\left(\tau_{i}\right)\right| \leq c_{3} \delta,
\end{gathered}
$$


where $b(t)=\ln V(t)$. Moreover,

$$
\begin{aligned}
& \left|\int_{\tau_{i}}^{t} a(\tau) C_{D O}(\tau) d \tau-\left(b\left(\tau_{i+1}\right)-b\left(\tau_{i}\right)\right) \xi_{i}^{h}\right| \\
& \quad \leq\left|\int_{\tau_{i}}^{t} a(\tau) C_{D O}\left(\tau_{i}\right) d \tau-\left(b\left(\tau_{i+1}\right)-b\left(\tau_{i}\right)\right) \xi_{i}^{h}\right|+\left|\int_{\tau_{i}}^{t} a(\tau)\left(C_{D O}(\tau)-C_{D O}\left(\tau_{i}\right)\right) d \tau\right| \\
& \quad \leq c_{4} \delta^{2}+\left|\left(b(t)-b\left(\tau_{i}\right)\right) C_{D O}\left(\tau_{i}\right)-\left(b\left(\tau_{i+1}\right)-b\left(\tau_{i}\right)\right) \xi_{i}^{h}\right| \\
& \quad \leq c_{4} \delta^{2}+c_{5} h+\left|b(t)\left(C_{D O}\left(\tau_{i}\right)-\xi_{i}^{h}\right)\right|+\left|b\left(\tau_{i+1}\right)-b(t)\right|\left|\xi_{i}^{h}\right| \leq c_{6}(h+\delta) .
\end{aligned}
$$

From inequality (21) we obtain

$$
\left|\tilde{w}_{0}^{h}(t)-C_{D O}(t)\right| \leq c_{7}(h+\delta+\alpha), \quad t \in T .
$$

From (32)-(36) in turn we obtain the inequality

$$
\sup _{t \in T}\left|\int_{t_{0}}^{t}\left(\varphi^{h}(\tau)-O U R(\tau)\right) d \tau\right| \leq c_{8}(h+\delta+\alpha) .
$$

Using (22) we obtain

$$
\begin{aligned}
\int_{t_{0}}^{\vartheta}\left|\varphi^{h}(\tau)-O U R(\tau)\right|^{2} d \tau & =\int_{t_{0}}^{\vartheta}\left|\varphi^{h}(\tau)\right|^{2} d \tau-2 \int_{t_{0}}^{\vartheta} \varphi^{h}(\tau) O U R(\tau) d \tau+\int_{t_{0}}^{\vartheta}|O U R(\tau)|^{2} d \tau \\
& \leq 2 \int_{t_{0}}^{\vartheta}\left(O U R(\tau)-\varphi^{h}(\tau)\right) O U R(\tau) d \tau+c_{9}(h+\delta) \alpha^{-1} .
\end{aligned}
$$

By the condition of the theorem, $\mu_{\max }(\cdot)$ is a function of bounded variation. Thus, $O U R(t)$ is also a function of bounded variation.

From (37), (38) and Lemma 1.3.3. [13] we thus obtain

$$
\int_{t_{0}}^{\vartheta}\left|\varphi^{h}(\tau)-O U R(\tau)\right|^{2} d \tau \leq c_{10} \lambda(h, \delta, \alpha) .
$$

The assertion of the theorem follows from (39) and Lemmas 4, 5. Q.E.D. 

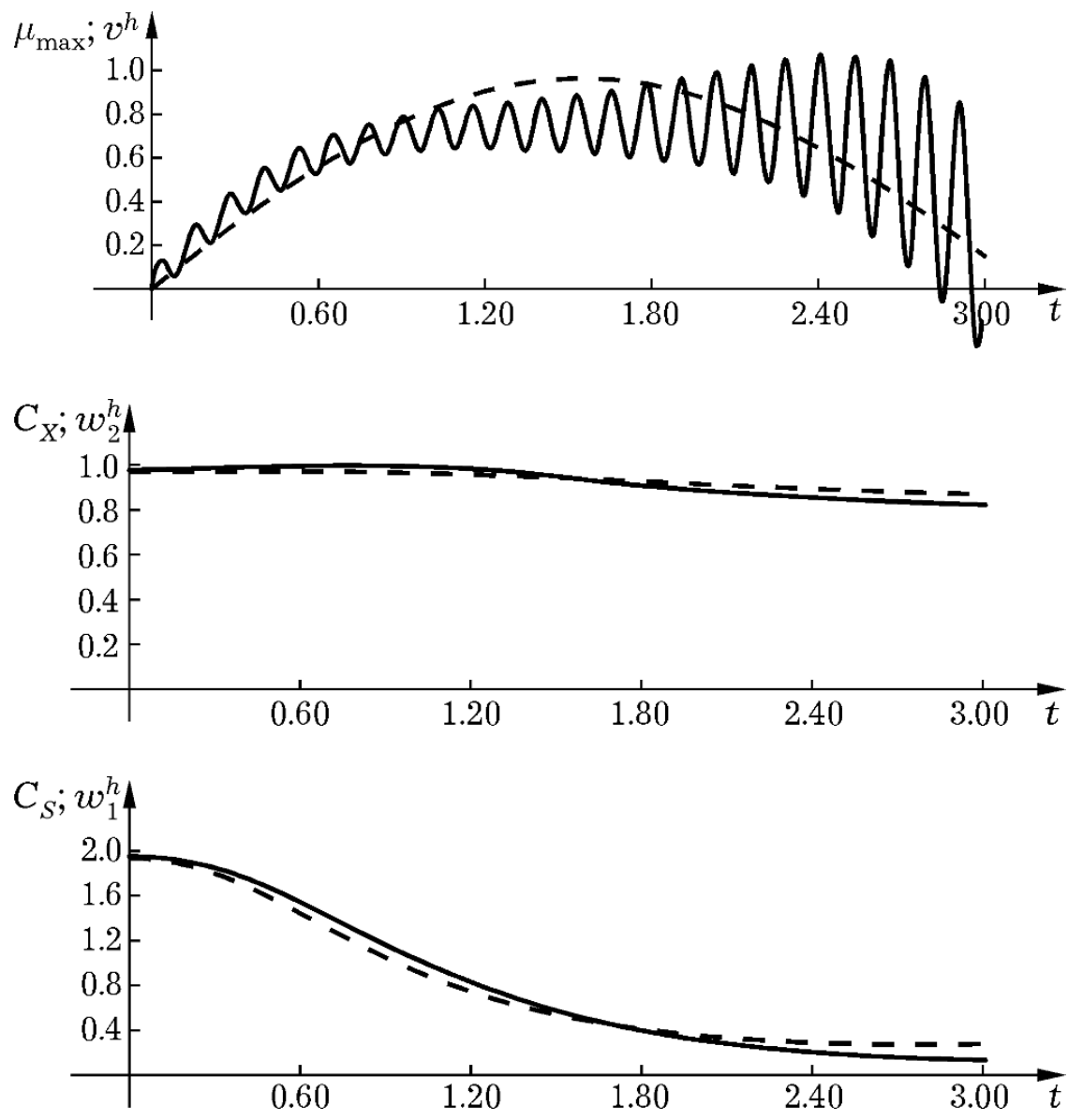

Fig. 1

Theorem 3 leads to the following corollary.

Corollary 1. Let $\delta(h)=h, \alpha(h)=h^{1 / 2}$ and assume that $\mu_{\max }(t)$ is a function of bounded variation. Then we have the following rate of convergence bounds for the algorithm:

$$
\begin{gathered}
\int_{t_{0}}^{\vartheta}\left|v^{h}(\tau)-\mu_{\max }(\tau)\right|^{2} d \tau \leq c_{1} h^{1 / 4}, \\
\sup _{t \in T}\left|w_{1}^{h}(t)-C_{X}(t)\right| \leq c_{2} h^{1 / 8}, \\
\sup _{t \in T}\left|w_{2}^{h}(t)-C_{S}(t)\right| \leq c_{3} h^{1 / 8} .
\end{gathered}
$$

Another corollary is valid for the case $F_{\text {in }}(t) \equiv 0$. Assume that the following condition holds instead of Condition 1. 

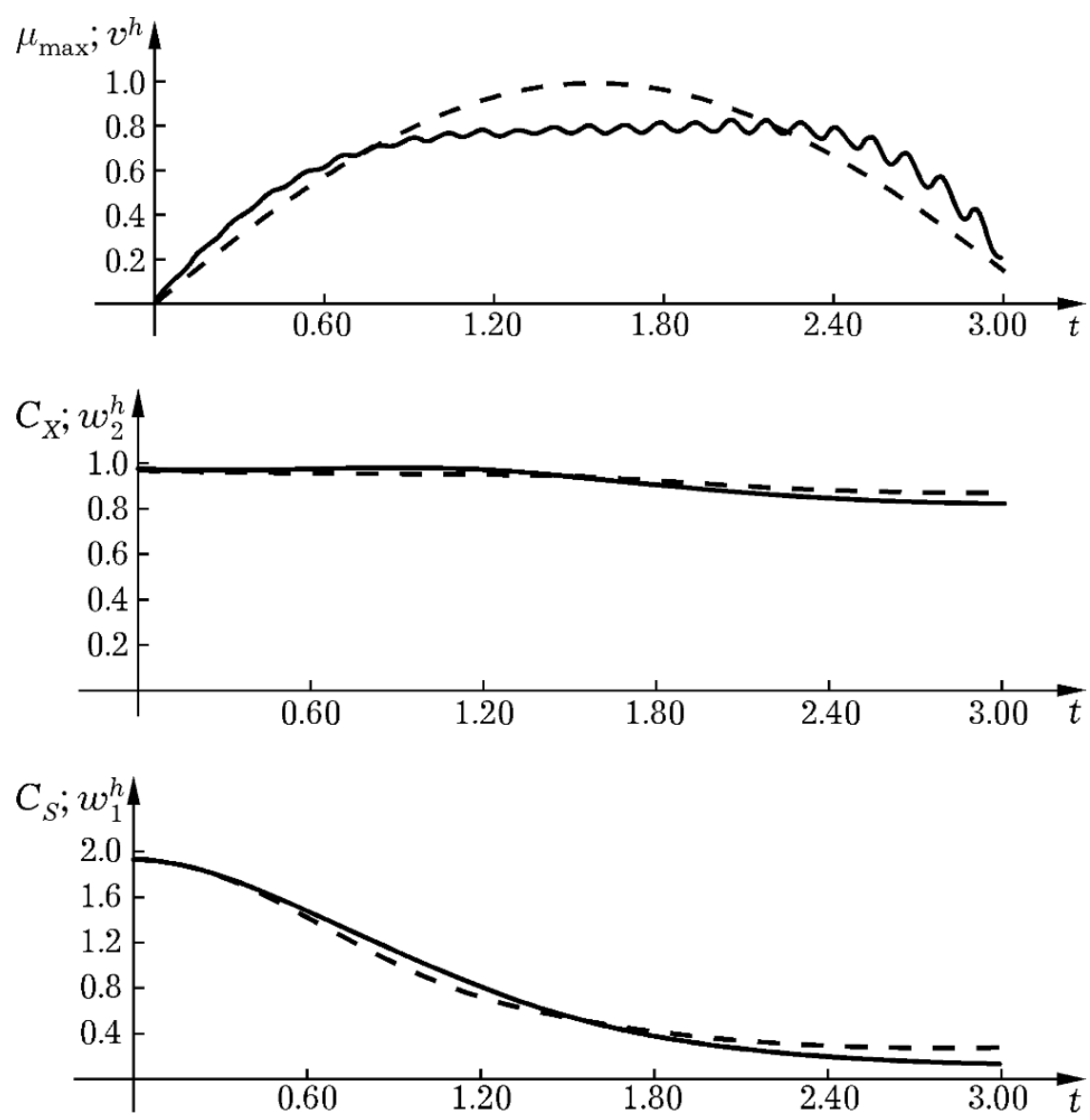

Fig. 2

\section{Condition 3.}

$$
\begin{gathered}
F(t)=0, \quad Y \in(0,1) \\
C_{X}(t) \geq C_{X}>0, \quad C_{S}(t) \geq C_{S}>0 .
\end{gathered}
$$

Then the second and third equations of system (1) take the form

$$
\begin{gathered}
\frac{d C_{X}(t)}{d t}=\frac{Y}{1-Y} \operatorname{OUR}(t), \\
\frac{d C_{S}(t)}{d t}=-\frac{1}{1-Y} \operatorname{OUR}(t) .
\end{gathered}
$$

Therefore, system $M$ may be taken in the following form:

$$
w_{0}^{h}\left(\tau_{i+1}\right)=w_{0}^{h}\left(\tau_{i}\right)+\delta\left\{k_{L a}\left(C_{\mathrm{sat}}^{\mathrm{en}}-\xi_{i}^{h}\right)-\varphi_{i}^{h}\right\},
$$



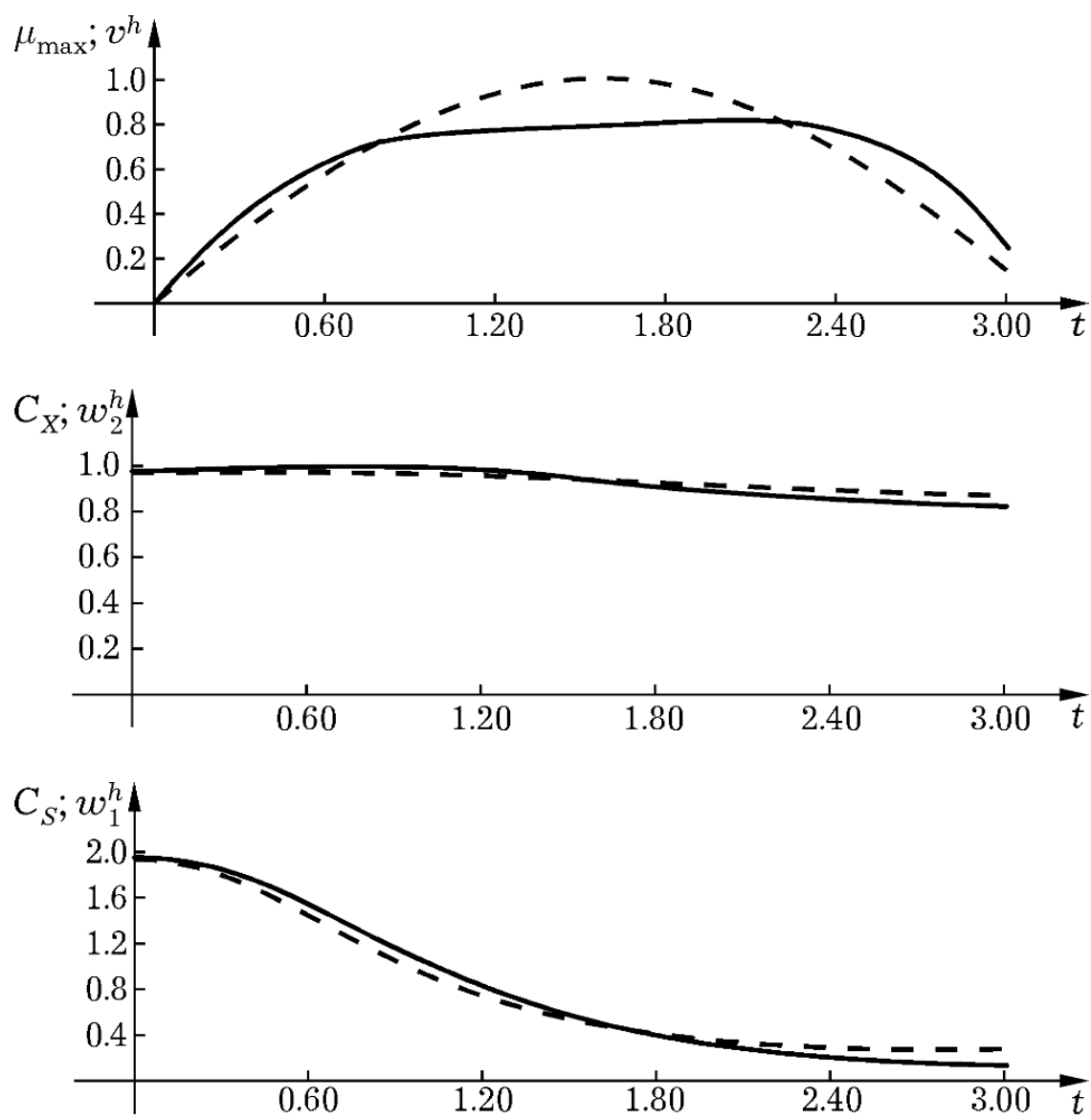

Fig. 3

$$
\begin{aligned}
& w_{1}^{h}\left(\tau_{i+1}\right)=w_{1}^{h}\left(\tau_{i}\right)+\delta \frac{Y}{1-Y} \varphi_{i}^{h}, \\
& w_{2}^{h}\left(\tau_{i+1}\right)=w_{2}^{h}\left(\tau_{i}\right)-\delta \frac{1}{1-Y} \varphi_{i}^{h} .
\end{aligned}
$$

The next assertion follows from the results given above.

Corollary 2. Assume that Conditions 2 and 3 hold, system $M$ has the form (40), and the function $\varphi^{h}(t)$ is given by (6). Then the assertions of Theorems 1-3 and Corollary 1 are true.

\section{Computer Simulation Results}

The algorithm described above has been tested on a prototype example. System (1) was considered on the time interval $[0,3]$. The coefficient $\mu_{\max }(t)$ was taken equal to $\sin (t)$. We assumed that $\mu_{\max }(t)$ was unknown and our objective was to reconstruct it. We also aimed to reconstruct the states $C_{X}(t)$ and $C_{S}(t)$ from finitely many observations of the evolution of the dissolved oxygen concentration in the reactor $C_{D O}(t)$.

Figures 1-3 plot the simulation results. The following system parameters were used (see (1)):

$$
K_{S}=0.1, \quad Y=0.5,
$$




$$
\begin{gathered}
C_{S, \text { in }}=0.2, \quad V\left(t_{0}\right)=1.5, \\
F_{\text {in }}(t)=\sin (t), \quad C_{\text {sat }}^{\text {en }}=C_{\text {sat }}=0.5 .
\end{gathered}
$$

The algorithm parameters were taken as $\alpha=0.001$ and $K=2$. The initial state of the system was set equal to 1 , i.e.,

$$
C_{D 0}\left(t_{0}\right)=C_{X}\left(t_{0}\right)=C_{S}\left(t_{0}\right)=1
$$

System (1) was solved by Euler's method with increment $\delta$. The simulation results demonstrate mean-square convergence of $v^{h}(t)$ to $\mu_{\max }(t)$ and uniform convergence of $w_{1}^{h}(t)$ to $C_{X}(t)$ and $w_{2}^{h}(t)$ to $C_{S}(t)$ for appropriate values of the parameters $\alpha, h$, and $\delta$.

Figure 1 corresponds to the case when $h=10^{-3} \cos (50 t), \delta=10^{-3}$; in Fig. $2, h=10^{-4} \cos (50 t)$, $\delta=10^{-3}$; and Fig. 3, $h=0, \delta=10^{-4}$. In Figs. $1-3$ the solid curve plots the coefficient $\mu_{\max }(t)$ and the states $C_{X}(t), C_{S}(t)$; the broken curve corresponds to the model control $v^{h}(t)$ and the auxiliary system coordinates $w_{1}^{h}(t)$ and $w_{2}^{h}(t)$.

The research has been carried out with the support of IIASA - International Institute of Applies System Analysis. The second author also acknowledges support from the Russian Foundation of Basic Research (grant 06-01-00359), the Program for the Support of Basic Research of the Presidium of the Russian Academy of Sciences "Control Processes," and the Program for the Support of Leading Scientific Schools in Russia (grant NSh7581.2006.1).

\section{REFERENCES}

1. A. N. Tikhonov and V. Ya. Arsenin, Methods of Solution of Ill-Posed Problems [in Russian], Nauka, Moscow (1978).

2. M. M. Lavrent'ev, V. G. Romanov, and S. P. Shishatskii, "Ill-Posed Problems of Mathematical Physics and Analysis," Transl. Math. Monographs, 93, AMS, Providence, RI (1986).

3. H. T. Banks and K. Kunisch, Estimation Techniques for Distributed Parameter Systems, Birkhäuser, Boston (1989).

4. Yu. S. Osipov and A. V. Kryazhimskii, Inverse Problems for Ordinary Differential Equations: Dynamical Solutions, Gordon and Breach, London (1995).

5. V. I. Maksimov, Dynamical Inverse Problems for Distributed Systems, VSP, Utrecht (2002).

6. A. V. Kryazhimskii, V. I. Maksimov, and Yu. S. Osipov, "Reconstruction of boundary sources through sensor observation," IIASA Working Paper WP-96-97, Ladenburg (1996).

7. A. V. Kryazhimskii and V. I. Maksimov, "On identification of nonobservable contamination inputs," Environmental Modeling and Software, 20, 1057-1061 (2005).

8. N. N. Krasovskii and A. I. Subbotin, Positional Differential Games [in Russian], Nauka, Moscow (1974).

9. P. A. Vanrolleghem and M. Van Daele, "Optimal experimental design for structure characterization of biodegradable models: on-line implementation in a respirographic biosensor," Water Science and Technology, 30, No. 4, 243-253 (1994).

10. J. D. Stigter, D. Vries, and K. J. Keesman, “On adaptive optimal input design,” Proc. European Control Conference, Cambridge 2003, Paper 066, Cambridge Univ. (2003).

11. K. J. Keesman, "State and parameter estimation for biotechnical batch reactors," Cont. Eng. Pract., 10, No. 2, 219-225 (2002). 